\title{
GPU Accelerated Non-rigid Registration for the Evaluation of Cardiac Function
}

\author{
Bo $\mathrm{Li}^{1}$, Alistair A. Young ${ }^{1,2}$, and Brett R. Cowan ${ }^{2}$ \\ ${ }^{1}$ Auckland Bioengineering Institute, University of Auckland, Auckland, New Zealand \\ \{b.1i, a.young\}@auckland.ac.nz \\ ${ }^{2}$ Auckland MRI Research Group, University of Auckland, Auckland, New Zealand \\ b. cowan@auckland.ac.nz
}

\begin{abstract}
We present a method for the fast and efficient tracking of motion in cardiac magnetic resonance (CMR) cines. A GPU accelerated LevenbergMarquardt non-linear least squares optimization procedure for finite element non-rigid registration was implemented on an NVIDIA graphics card using the OpenGL environment. Points were tracked from frame to frame using forward and backward incremental registration. The inner (endocardial) and outer (epicardial) boarders of the heart were tracked in six short axis cines with $\sim 25$ frames through the cardiac cycle in 36 patients with vascular disease. Contours placed by two independent expert observers using a semi-automatic ventricular analysis program (CIM version 4.6) were used as the gold standard. The method took 0.5 seconds per frame, and the maximum Hausdorff errors were less than 2 $\mathrm{mm}$ on average which was of the same order as the expert inter-observer error. In conclusion, GPU accelerated Levenberg-Marquardt non-linear optimization enables fast and accurate tracking of cardiac motion in CMR images.
\end{abstract}

Keywords: Graphics Processing Unit (GPU), Image Registration, Cardiac Magnetic Resonance Imaging (CMR).

\section{Introduction}

Cardiac magnetic resonance (CMR) imaging provides an abundant source of detailed, quantitative data for the accurate evaluation of the structure and function of the heart [1]. Advantages of CMR include its non-invasive nature, ability to modulate tissue contrast, lack of radiation, and ability to provide high quality functional information in any arbitrary imaging plane.

However, image processing for quantitative analysis is complex and timeconsuming. For example, the epicardial (the interface between the outer surface of the heart muscle and adjacent structures) and endocardial (interface between the inner surface of the heart muscle and blood) contours are typically segmented by expert, highly trained clinicians or analysts [2] assisted by semi-automated software, and analysis of the many hundreds of images represents a significant bottleneck. This problem can be improved using automated tracking techniques such as optical flow [3] or non-rigid registration [4]. The latter maps images to a deformable model and 
maximizes a similarity measure between the current image and reference image by modifying the parameters of the model in order to give an estimate of the underlying motion.

Recent advances in the graphics processing units (GPU) available on standard low price personal computers provide additional power for general-purpose computation [5]. The work of Strzodka et al. is one of the earliest to use the programmable floating point capacities of the graphics hardware in image registration [6]; they use an efficient multi-grid representation of the image multi-scale, and an adaptive time-step control of the iterative solvers to achieve fast registration.

In this paper, we present a GPU-based non-rigid image registration method for cardiac motion estimation, which uses the sum of squared (SSD) pixel intensity differences as the similarity measure and the Levenberg-Marquardt non-linear least squares algorithm for optimization. Novel aspects of our work include: a novel finite element (FE) registration procedure incorporating a flexible global-to-local parameter map, image warping in reference space to allow pre-calculation of finite element basis functions, an optimized GPU algorithm taking advantage of the local support of finite element basis functions, and GPU calculation of the value, gradient and Hessian of the objective function. Validation was performed by comparison with two independent expert analysts in patient data from a clinical trial. The errors between the algorithm and each analyst were found to be comparable to the differences between the analysts. We also achieved significant improvements in speed compared to an equivalent CPU implementation, and a standard GPU gradient descent registration algorithm.

\section{Method}

The method was implemented using a region of interest (ROI) typically comprising $128 \times 128$, square grey-scale pixels. Assuming the current and reference images are $I_{t}$, $I_{0}$, let the reference coordinates be $\boldsymbol{X}$ and the current coordinates be $\boldsymbol{x}$. A FE description of the displacement field, $\boldsymbol{u}$, controlled by a regular global lattice of control points $\boldsymbol{P}$, was created over the ROI, in order to define the mapping between frames as the displacement from reference to current coordinates:

$$
\boldsymbol{x}(\boldsymbol{X})=\boldsymbol{X}+\boldsymbol{u}(X)
$$

A flexible global-to-local parameter map was used to link the global lattice $\boldsymbol{P}$ to the local lattices, $\boldsymbol{P}^{e}$, of each element, as defined by $\boldsymbol{u}(\boldsymbol{X})=\sum \psi^{e}(\boldsymbol{X}) \boldsymbol{P}^{e}$. Bi-cubic Bézier basis functions with $\mathrm{C} 1$ continuity between the elements were used. Image warping of the current image according to $\boldsymbol{u}$ takes place in reference space with respect to $\boldsymbol{X}$ : $M_{\mathrm{t}}(\boldsymbol{X})=I_{\mathrm{t}}(\boldsymbol{x}(\boldsymbol{X}))$, so that the warped current image, $M_{\mathrm{t}}$, is generated by mapping the current image to the reference coordinates. Advantages of using C1 Bézier basis functions include: (1) each global control point has very local support in that only the deformation within the four elements around the node are affected, and (2) a multi grid optimization can be performed using de Casteljau's algorithm.

The similarity measure, or objective function of the registration, was the SSD of pixel intensities (scaled to $[0,1]$ ) in the ROI between the warped current image $M_{t}$ and reference image $I_{0}$ in reference coordinates: 


$$
E_{I}=\sum w^{2}(\boldsymbol{X})\left(I_{0}(\boldsymbol{X})-M_{\mathrm{t}}(\boldsymbol{X})\right)^{2}
$$

where $w^{2}(\boldsymbol{X})$ is a weight image included to enable different weighting in different parts of the image. In practice the image data and FE model may not be sufficient to regularize the problem, which is ill-posed in the sense of Hadamard. A Sobolev smoothing term [7] is therefore included in the error function:

$$
E_{S}=\int_{\Omega} \alpha_{1}\left\|\frac{\partial \mathbf{u}}{\partial X}\right\|^{2}+\alpha_{2}\left\|\frac{\partial \mathbf{u}}{\partial Y}\right\|^{2}+\beta_{1}\left\|\frac{\partial^{2} \mathbf{u}}{\partial X^{2}}\right\|^{2}+\beta_{2}\left\|\frac{\partial^{2} \mathbf{u}}{\partial Y^{2}}\right\|^{2}+\beta_{3}\left\|\frac{\partial^{2} \mathbf{u}}{\partial X \partial Y}\right\|^{2} d \Omega
$$

where $\alpha_{1}, \alpha_{2}, \beta_{1}, \beta_{2}$ and $\beta_{3}$ are smoothing weights. The integration is performed over $\Omega$, the domain of the registration.

The gradient of the image term with respect to model parameter $P_{i}$ is:

$$
\begin{aligned}
& g_{i}=-2 w^{2}(X)\left(I_{0}(X)-M_{t}(X)\right) \frac{\partial M_{t}(\mathbf{X})}{\partial P_{i}} \\
& \frac{\partial M_{t}(\mathbf{X})}{\partial P_{i}}=\frac{\partial I_{t}(\mathbf{x}(\mathbf{X}))}{\partial P_{i}}=\left.\frac{\partial I_{t}(\mathbf{x})}{\partial \mathbf{x}}\right|_{\mathbf{x}=\mathbf{X}(\mathbf{X})} \cdot \frac{\partial \mathbf{x}}{\partial P_{i}}=\left.\frac{\partial I_{t}(\mathbf{x})}{\partial \mathbf{x}}\right|_{\mathbf{x}=\mathbf{X}(\mathbf{X})} \cdot \frac{\partial \mathbf{u}(X)}{\partial P_{i}}=\left.\frac{\partial I_{t}(\mathbf{x})}{\partial \mathbf{x}}\right|_{\mathbf{x}=\mathbf{X}(\mathbf{X})} \cdot \Psi_{i}(X)
\end{aligned}
$$

showing that $\frac{\partial M_{t}(\mathbf{X})}{\partial P_{i}}$ is the product of the warped gradient or edge image of the current image with the basis function map $\psi(\mathrm{X})$.

The Hessian of the image term is approximated by [7]:

$$
H_{i j}=2 w^{2}(X) \frac{\partial M_{t}(\mathbf{X})}{\partial P_{i}} \frac{\partial M_{t}(\mathbf{X})}{\partial P_{j}}
$$

With the known gradient and Hessian image and smoothing terms, the objective function $E=E_{I}+E_{S}$ is optimized using the Levenberg-Marquardt algorithm, as described in [8] At each iteration, a system of linear equations is solved using the conjugate gradient algorithm.

In order to track the motion throughout multiple time frames, a multi-level method [4] is used to sum the deformations at previous frames: $x(X, t)=x_{\mathrm{t}-1} \ldots x_{0}(X)$. Thus, after registering the image at frame 1 to $0\left(x_{0}\right)$ we use the registration from frame 2 to $1\left(x_{1}\right)$ as a second level to warp the result of $x_{0}$. A potential problem for this tracking method is that once a tracking error occurs at a frame, it will be propogated to all subsequent frames. To ameliorate this problem, we performed two separate registrations along the forward $x^{\mathrm{f}}$ and backward $x^{\mathrm{b}}$ time directions thought the cardiac cycle, and interpolated forward and backward results by:

$$
\boldsymbol{x}_{\mathbf{t}}(\boldsymbol{X})=(1-w) x^{\mathrm{f}}(\boldsymbol{X})+w x^{\mathrm{b}}(\boldsymbol{X}) \quad \text { where } w=\mathrm{t} /(\text { total number of frames })
$$

This method (although simple) ensures periodicity and enforces continuity between the beginning and end of the cardiac cycle with the first and last frames. 


\section{GPGPU Implementation}

The implementation of non-rigid image registration can be divided into CPU and GPU components (as shown in Fig.1). There are three major tasks in the CPU: (1) off-line and once only pre-computation of the Standard Basis Texture (texture of basis functions) and Current Edge Image, (2) re-arrangement of the pixels in the Gradient Texture and Hessian Texture returned from the off-screen frame buffer into the gradient vector (Eqn.4) and Hessian matrix (Eqn.5), followed by solution using the Conjugate Gradient Solver, (3) checking the convergence of the current solution based on the similarity measure, which is returned in the green pixels of the Warped Texture. Before the GPU begins, the global parameters are encoded in a texture, in which each row represents an element of the model and contains its corresponding parameters. The current and reference images, Parameter Texture and the Standard Basis Texture are transferred to the GPU memory by RGBA textures with 32 bit floating point support.

In the GPU, three fragment shaders (Warping Shader, Gradient Shader and Hessian Shader) execute the highly computational components of the registration: error function, gradient and Hessian calculation respectively.

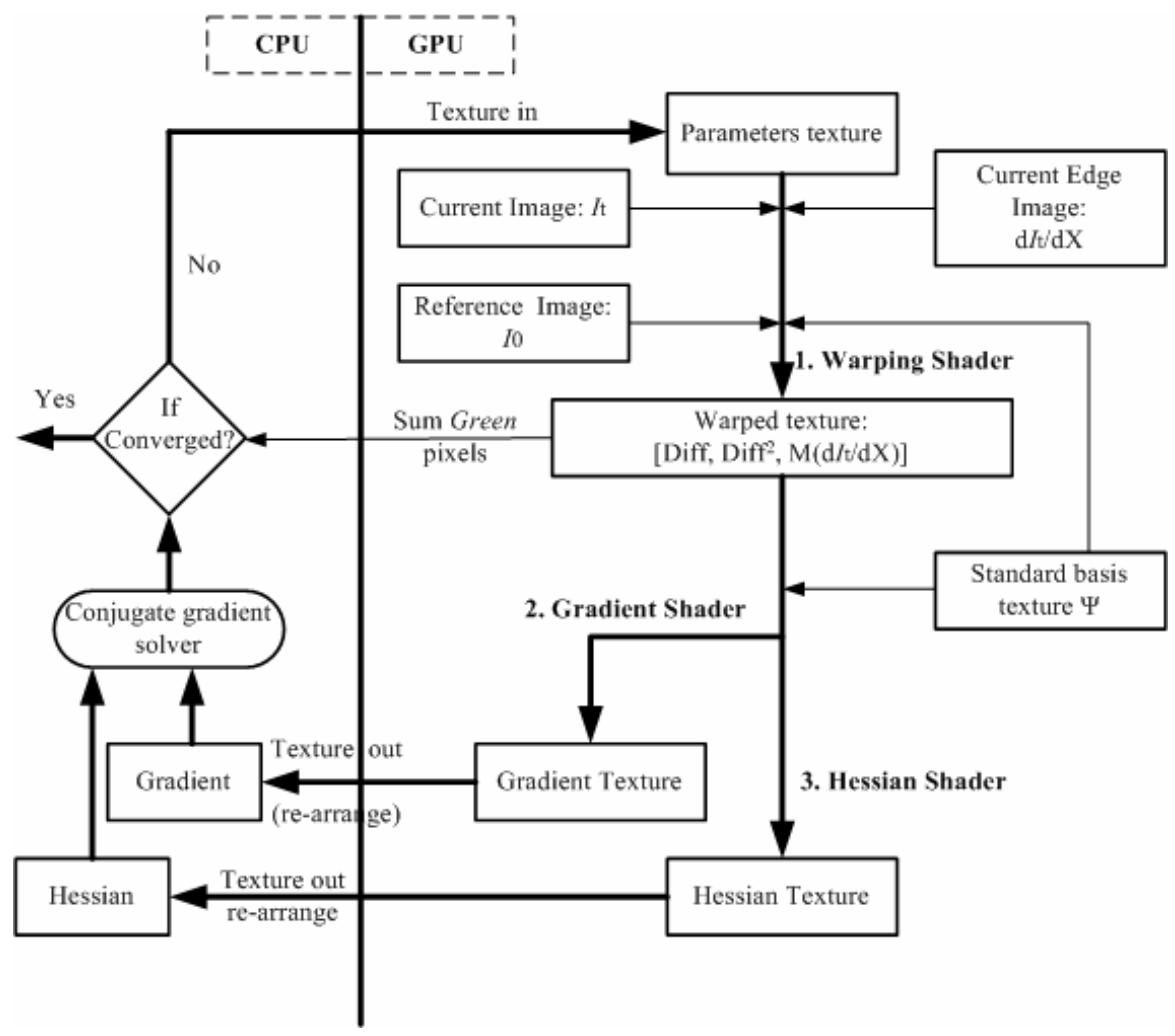

Fig. 1. Schema for the GPU implementation of non-rigid image registration 
Prior to execution of the Warping Shader, the graphics viewport must be set to the dimensions of the image in order to ensure the correct number of pixels are rendered. The element coordinates for the rendered region $\left(\xi_{1}, \xi_{2}\right)$ must also be passed through the OpenGL texture coordinates, so that each fragment is accurately mapped to a pixel on the reference image.

Inside the Warping Shader, each fragment uses the $\left(\xi_{1}, \xi_{2}\right)$ from the texture coordinates to find its corresponding 16 parameters in Parameter Texture and 16 basis functions in the Standard Basis Texture using texture lookup (16 basis functions are returned in the RGBA channels of 4 pixels). Summing the product of the returned parameters and the basis functions provides the warped position for the current fragment, $M\left(\xi_{1}, \xi_{2}\right)$. Finally, the Warping Shader uses this warped position to asynchronously calculate additional information and place this into the RGBA channels of the rendering texture. These are:

R: The pixel difference between reference and warped current image:

G: The squared pixel difference: Diff ${ }^{2}$

$$
\operatorname{Diff}=I_{0}\left(\xi_{1}, \xi_{2}\right)-I_{\mathrm{t}}\left(M\left(\xi_{1}, \xi_{2}\right)\right)
$$

$\mathbf{B}, \mathbf{A}$ : The warped Current Edge Image in reference space $\left.\frac{\partial I_{t}(\mathbf{x})}{\partial \mathbf{x}}\right|_{\mathbf{x}=\mathbf{x}(\mathbf{X})}$

This rendered texture is used as the input texture to the shaders for gradient, Hessian, and error function calculation (sum of the green pixels).

As shown in Eqn.4, the gradient of the objective function with respect to each parameter $P_{\mathrm{i}}$ can be derived from the pixel-wise summation of the product of following three images:

- The difference image between $I_{0}$ and $M_{\mathrm{t}}$ : Diff $=I_{0}-M_{\mathrm{t}}$,

- The Current Edge Image warped to the reference state $\left.\frac{\partial I_{t}(\mathbf{x})}{\partial \mathbf{x}}\right|_{\mathbf{x}=\mathbf{x}(\mathbf{X})}$, and

- The basis function map with respect to each parameter $P_{\mathrm{i}}: \psi_{\mathrm{i}}$.

The difference image and the warped Current Edge Image are already known from the previous step. Since 16 basis function maps are required by all pixels, the product of the images requires an output region which is 4 times larger than the original image (RGBA channels are used). In the Gradient Shader, a region four times larger is rendered. Each fragment within this region contains four basis functions required by its corresponding pixel, and calculates the product of the above terms in parallel (RGBA channels). Then, an additional fragment shader, which implements reduction [5], is used to efficiently calculate the sum of all pixels within each element in order to finally generate the Gradient Texture in Fig.1.

The Hessian Texture is generated in the similar manner as the Gradient Texture, but is more complicated. Eqn.5 suggests that the Hessian requires the pixel-wise sum of the product of any two basis function maps: $\psi_{\mathrm{i}}$ and $\psi_{\mathrm{j}}$ and the squared warped Current Edge Image, a minimum of 136 basis function maps are required by all pixels. In the Hessian Shader, a region 36 times larger is rendered, each fragment of which calculates the product of the required terms. Also, another reduction fragment program is used to generate the Hessian Texture in the output texture. 


\section{Validation}

The GPU non-rigid registration procedure was applied to images from 36 patients with vascular disease chosen at random from the baseline studies of the ONTARGET MRI sub-study, as described in [11]. Briefly, either prospectively or retrospectively gated steady state free precession (SSFP) CMR cines were acquired in six equally spaced short axis (SA) locations from apex to base. Typical imaging parameters were $\mathrm{TR} / \mathrm{TE} / \mathrm{flip} / \mathrm{FOV}=30 \mathrm{~ms} / 1.6 \mathrm{~ms} / 60^{\circ} / 360 \mathrm{~mm}$, slice thickness $6 \mathrm{~mm}$, image matrix $256 \times 208$. There were typically 25 temporal cine frames per slice, depending on the heart rate. All cines were acquired during breath-holding of 8-15 seconds duration.

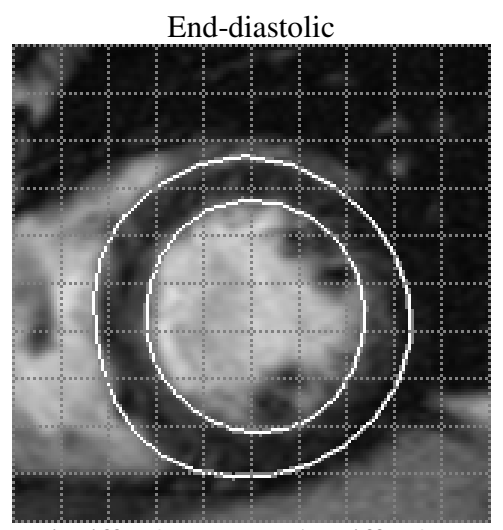

Epi Diff $=0 \mathrm{~mm} \quad$ Endo Diff $=0 \mathrm{~mm}$

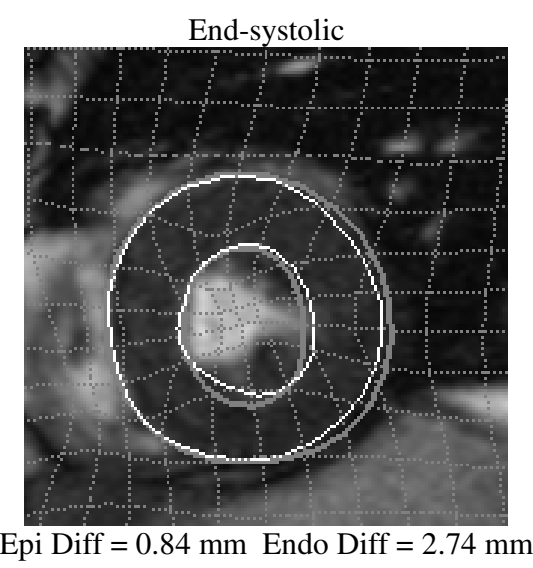

Fig. 2. Typical results based on end-diastolic frame (largest blood volume during the cardiac cycle) where the endocardial and epicardial manual and registered contours are identical. At end-systole (time of smallest blood volume), the manual (grey) and registered (light) contours are shown.

Epicardial and endocardial contours were defined on each image by two independent observers $(A$ and $B$ ) using guide point modelling [8]. In this semi-automated procedure, contours are defined and tracked throughout the cycle using a combination of user-placed guide points and image edge points. This method has been previously validated against animal autopsy studies, manual contouring in patients with regional wall motion abnormalities, and against independent measures of cardiac output in healthy volunteers [9].

In order to validate the non-rigid registration procedure for tracking purposes, ED and ES contours from each expert observer were tracked through the rest of the cardiac cycle using the multi-resolution procedure. Preliminary experiments (not shown) indicated optimal smoothing weights of $\alpha=0.001 \beta=0.0001$ in Eqn.3). The modified Hausdorff (HD) distance [10] was used to quantify errors between the automatically tracked contour and the expert observer contour (as shown in Fig.2). The errors between contours obtained from each observer (labelled A and B) were stable through all parts of the cardiac cycle, averaging $1.9 \mathrm{~mm}$ for the epicardium and $2.1 \mathrm{~mm}$ for the endocardium. These are used as an indication of the intrinsic variability of contour identification. 
Fig.3 shows the average Hausdorff distance between contours from each of the expert observers A and B and the automatically tracked contours started from the ED frame from each observer and propagated through the cardiac cycle by the algorithm. The results from each case were interpolated to give 25 generic "frames" for the purposes of result presentation. For both the epicardial (left) and endocardial (right) plots in Fig.3, the distance starts from zero at ED frame, in which the tracked contour is identical to the expert contour, and reach a maximum at the ES frame of about $2 \mathrm{~mm}$. For contours tracked starting at the ES frame, similar results (not shown) were obtained, but the minimum distance occurred at the ES frame and the maximum distance at the ED frame.
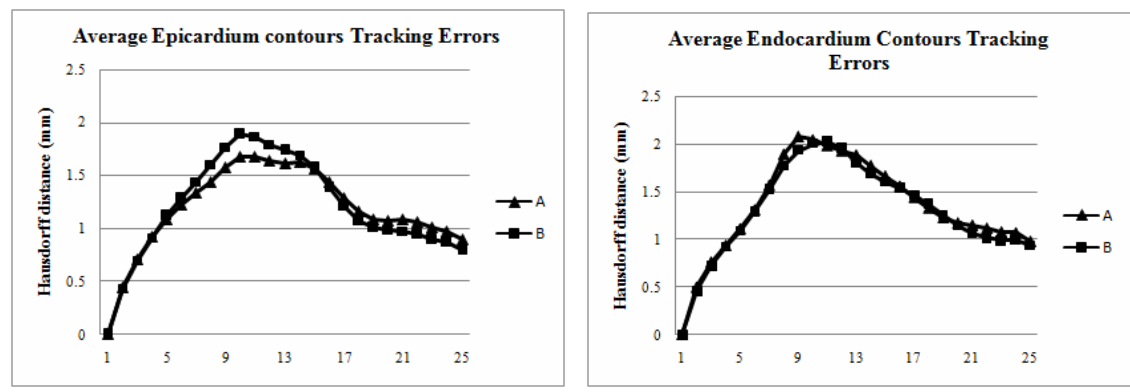

Fig. 3. Average modified Hausdorff distances between epicardial (left) and endocardial (right) contours between observers $A$ and $B$ and the automatically tracked contours started at the enddiastolic frame

The speed of the method was firstly compared against a fully CPU implementation and secondly a previously described [6] GPU registration using a gradient descent algorithm. Although each step in the GPU gradient descent is faster than the Levenberg-Marquardt step (all calculations occur in GPU), it takes many more steps to converge. The registration was applied to a randomly chosen patient with 6 slices, 19 temporal frames per slice and region of interest (ROI) of 128x128 (in total 380 registrations were required) using four different processors:

Table 1. Performance results. $(\mathrm{GD}=$ gradient descent; $\mathrm{LM}=$ Levenberg- Marquardt $)$. CPU: total time required for fully CPU implementation; GPU: total time required for GPU/CPU implementation.

\begin{tabular}{lccccc}
\hline & $\begin{array}{c}\text { Intel } 3.4 \\
\mathrm{GHz}\end{array}$ & $\begin{array}{c}\text { Intel dual } \\
\text { core } 2.0 \mathrm{GHz}\end{array}$ & $\begin{array}{c}\text { NVIDIA } \\
\text { 7700 Go }\end{array}$ & $\begin{array}{c}\text { NVIDIA } \\
\text { 7950 GTX }\end{array}$ & $\begin{array}{c}\text { NVIDIA } \\
\text { 7700 Go }\end{array}$ \\
\hline & $\mathrm{CPU}$ & $\mathrm{CPU}$ & GPU(LM) & GPU(LM) & GPU (GD) \\
\hline Speed (sec/frame) & 2.43 & 2.12 & 0.85 & 0.46 & 8.7
\end{tabular}

\section{Discussion and Future Work}

In this paper, a fully automated tracking process gave errors of the same order as the inter-observer error between expert analysts. In comparison to other non-rigid image 
registration techniques $[4,6]$, our approach reports a faster solution both in theory and practice. The gradient and Hessian calculation processes have fixed computational cost regardless of the complexity of the model. Comparison with a gradient descent algorithm showed much slower convergence than the Levenburg-Marquardt approach. The full port of similarity measure, gradient and Hessian calculations to the GPU allowed us make better use of the advanced graphics hardware to perform nonrigid registration calculations.

The limitations of our non-rigid image registration are that its performance depends mainly on the quality and the signal to noise ratio of the images. Also, in motion tracking, the tracking error existing between two consecutive frames is passed to the following frames and this can be hard to correct, so that tracked motions furthest from the starting phase contain the maximum tracking error.

In future work, we are planning to extend our current $2 \mathrm{D}$ non-rigid image registration to the time domain, by integrating time domain parameters into the deformable model. Also, migration to the NVIDIA CUDA technology will enable speed improvements, for example by implementing the conjugate gradient solver on the GPU.

\section{References}

1. Young, A.A.: Assessment of Cardiac Performance with Magnetic Resonance Imaging. Current Cardiology Reviews 2, 271-282 (2006)

2. Karamitsos, T.D., Hudsmith, L.E., Selvanayagam, J.B., Neubauer, S., Francis, J.: Operator induced variability in left ventricular measurements with cardiovascular magnetic resonance is improved after training. J. Cardiovasc. Magn. Reson. 9(5), 777-783 (2007)

3. Sundar, C.A., Hubert, J.V.: A new approach to study cardiac motion: The optical flow of cine MR images. Magnetic Resonance in Medicine 29(1), 59-67 (1993)

4. Chandrashekara, R., Mohiaddin, R.H., Rueckert, D.: Analysis of 3-D myocardial motion in tagged MR images using non-rigid image registration. IEEE Trans. Med. Imaging 23(10), 1245-1250 (2004)

5. Owens, J.D., Luebke, D., Govindaraju, N., Harris, M., Kruger, J., Lefohn, A.E., Purcell, T.J.: A survey of general-purpose computation on graphics hardware. Computer Graphics Forum 26(1), 80-113 (2007)

6. Strzodka, R., Droske, M., Rumpf, M.: Image registration by a regularized gradient flow.A streaming implementation in DX9 graphics hardware. In: Computing 2004, pp. 373-389 (2004)

7. Kass, M., Witkin, A., Terzopoulos, D.: Snakes: Active contour models. International Journal of Computer Vision 1, 321-331 (1987)

8. Press, W.H., Teukolsky, S.A., Vetterling, W.T., Flannery, B.: Non-linear Models. In: Numerical Recipes in C: The Art of Scientific Computing, $2^{\text {nd }}$ edn., ch. 15-5 (2002)

9. Young, A.A., et al.: Left ventricular mass and volume: Fast calculation with guide-point modeling on MR images. Radiology 216(2), 597-602 (2000)

10. Dubuisson, M.P., Jain, A.K.: A Modified Hausdorff Distance for Object Matching. Conference A: Computer Vision \& Image Process. In: Proc. 12th IAPR Internat. Conf. on Pattern Recogn., October 9-13, 1994, vol. 1, pp. 566-568 (1994)

11. Anderson, C.: Rationale and design of the cardiac magnetic resonance imaging substudy of the ONTARGET trial programme. J. Int. Med. Res. 33 (Suppl. 1), 50A-57A (2005) 